\title{
Short communication: Development of a direct in vivo screening model to identify potential probiotic bacteria using Caenorhabditis elegans
}

\author{
M. R. Park, H. S. Yun, S. J. Son, S. Oh, and Y. Kim ${ }^{1}$ \\ BK21 Plus Graduate Program, Department of Animal Science and Institute Agricultural Science \& Technology, Chonbuk National University, \\ Jeonju, 561-756, Korea
}

\begin{abstract}
Caenorhabditis elegans is an accepted model host to study host-bacteria interactions in the gut, in addition to being a simple model with which to study conserved aspects of biological signaling pathways in intestinal environments, because these nematode worms have similar intestinal cells to those of humans. Here, we used $C$. elegans to develop a new in vivo screening system for potential probiotic lactic acid bacteria (LAB). Initially, critical colonization ability of LAB strains isolated from Korean infant feces was screened in the worm intestinal tract over a period of $5 \mathrm{~d}$. Furthermore, we investigated host health-promoting activities, including longevityextending effects and immune-enhancing activities against foodborne pathogen infection. We identified 4 LAB strains that were highly persistent in the nematode gut and that significantly prolonged the longevity of $C$. elegans and improved the survival of $C$. elegans in response to infection by Staphylococcus aureus. The 4 LAB strains we identified showed resistance to acid and bile conditions, assimilated cholesterol, and were able to attach to a mucus layer. The $4 \mathrm{LAB}$ isolates were identified as Lactobacillus plantarum using $16 \mathrm{~S}$ rRNA sequencing analysis. Taken together, we developed a direct in vivo screening system using $C$. elegans to study host health-promoting LAB. Our system is simple, rapid, cost-effective, and reliable, and we anticipate that this system will result in the discovery of many more potential probiotic bacteria for dairy foods. Key words: probiotic, Caenorhabditis elegans, colonization, antiaging, immune response
\end{abstract}

\section{Short Communication}

Lactic acid bacteria (LAB) are generally recognized as safe and play an important role in food and feed fermentation (FAO/WHO, 2002). In addition, as probiotic bacteria, LAB benefit the host by improving its intestinal microbial balance as well as by providing im-

Received July 3, 2014.

Accepted July 26, 2014.

${ }^{1}$ Corresponding author: ykeys2584@jbnu.ac.kr munomodulatory effects (Fuller, 1989). In recent years, interest in the host health-promoting effects of probiotics has increased. As a result, numerous probiotic products have been developed to improve health (Heller, 2001). Many research groups are actively investigating novel probiotics as well as the functional characteristics of potential probiotic candidates. Numerous in vitro tests to assess factors such as survival ability during gastrointestinal transit (acid tolerance/bile resistance), intestinal epithelial adhesion properties, and antimicrobial substance-producing abilities have been developed for preliminary identification of potential probiotic LAB strains (Sanders, 2008; Turchi et al., 2013). Superior probiotic candidates have desirable physiological properties such as immunomodulation, cholesterol reduction, antimutagenic, and anticarcinogenic activities (du Toit et al., 1998; Guo et al., 2010). However, in vivo studies in animal and human hosts are required to establish safety and efficacy (FAO/WHO, 2002).

As noted above, many different criteria have been used to select potential probiotic strains. Of these, probiotic attachment to intestinal epithelial cells and colonization of the intestinal tract are the most important factors because these affect the ability of the bacterial species to function as a probiotic and are important for maintenance of the normal gut microbiota balance in the host (Ouwehand et al., 1999). Thus, most studies to select strains that can adhere to the epithelium surface have focused on alternative in vitro models, such as propagated human intestinal cell lines (e.g., HT-29 and Caco-2 cells) because of difficulties experienced in studying bacterial adhesion in vivo, especially in humans (Duary et al., 2011). Recently, specific target hosts, including Artemia nauplii (brine shrimp) and Cyprinus carpio (common carp), have been used to validate or screen probiotic potential (Sugimura et al., 2011; Lamari et al., 2014). Unfortunately, these organisms have critical limitations with regard to reflecting the human gut. We therefore developed a new in vivo system to screen potential probiotic bacteria for host health-promoting activities.

Caenorhabditis elegans as a model system for screening has several practical advantages: screening is easy 
to perform, rapid, low-cost, and can be scaled up. Furthermore, the body of this nematode is transparent, allowing clear observation of all cells in mature and developing animals (Leung et al., 2008). More importantly, C. elegans has intestinal cells that are similar in structure to human intestinal cells (McGhee, 2007). Therefore, our objective in this study was to develop a simple, rapid, and economic high-throughput screening method for potential probiotic bacteria using $C$. elegans as an in vivo model.

Approximately 2,000 LAB stains were isolated from the feces of breast-feeding infants under 9 mo of age from a postnatal care center in Jeonju (Jeollabuk-do, Korea). Five grams of fecal sample was diluted in 45 $\mathrm{mL}$ of L-cysteine solution $(8.5 \mathrm{~g}$ of $\mathrm{NaCl}, 0.5 \mathrm{~g}$ of $\mathrm{L}-$ cysteine, and $1 \mathrm{~L}$ of distilled water) and plated on de Man, Rogosa, and Sharpe (MRS)-bromocresol purple $(0.004 \%)$ agar at $37^{\circ} \mathrm{C}$ for $48 \mathrm{~h}$. Yellow single colonies were selected randomly, subcultured in MRS broth, and restreaked onto MRS agar to ensure purity. Basic assays such as Gram staining and catalase tests were performed according to Buchanan and Gibbons (1974). All isolated $\mathrm{LAB}$ were maintained at $-80^{\circ} \mathrm{C}$ in MRS broth containing glycerol solution for further studies. All strains were subcultured $\left(\sim 1 \times 10^{9} \mathrm{cfu} / \mathrm{mL}\right) 3$ times before use. The $C$. elegans strain used in this study was CF512 fer-15(b26)II;fem-1(hc17)IV (Kim and Mylonakis, 2012). This C. elegans strain was routinely maintained on nematode growth medium (NGM) plates seeded with Escherichia coli OP50, as described previously (Brenner, 1974).

First, to screen for colonization of the C. elegans intestinal tract, the number of bacterial cells in worm intestines were measured according to previously described methods, with slight modifications (Garsin et al., 2001). After exposing C. elegans to individual probiotic candidates on NGM plates containing nystatin $(5 \mu \mathrm{g} / \mathrm{mL})$ for $5 \mathrm{~d}, 10$ worms were picked randomly, washed twice in M9 buffer, and placed on brain-heart infusion plates containing both kanamycin $(100 \mu \mathrm{g} /$ $\mathrm{mL})$ and streptomycin $(100 \mu \mathrm{g} / \mathrm{mL})$. These plates were exposed to $5 \mu \mathrm{L}$ of gentamicin solution $(25 \mu \mathrm{g} / \mathrm{mL})$ for $5 \mathrm{~min}$, and results were compared with Lactobacillus rhamnosus GG as a positive control. Next, worms were washed 5 times with M9 buffer, transferred into a 1.5$\mathrm{mL}$ Eppendorf tube containing M9 buffer with 1\% Triton X-100, and mechanically disrupted using a pestle (Kontes Glass Inc., Vineland, NJ). These diluted worm lysates were plated on modified MRS ( $\mathrm{pH} 5.0$ ) agar and incubated at $37^{\circ} \mathrm{C}$ for $48 \mathrm{~h}$. In addition, to determine LAB colonization in the $C$. elegans intestine, we used transmission electron microscopy (H-7650; Hitachi Co. Ltd., Tokyo, Japan) of the intestinal epithelium to evaluate their persistence at $5 \mathrm{~d}$. For each observation, at least 5 cross sections were evaluated whenever possible, and representative images were chosen.

Next, to evaluate whether the probiotic candidates had an effect on host lifespan, nematode lifespan was measured according to previously described methods with modifications (Aballay et al., 2000; Garigan et al., 2002). Twenty worms were exposed to probiotic candidates on NGM plates and the numbers of live worms were scored every day. To correct the score, worms were transferred to fresh plates every $3 \mathrm{~d}$. A worm was regarded as dead when it failed to respond to gentle touch. Then, to estimate whether the probiotics influence on the resistance of host against the infection of foodborne pathogens, killing assays were conducted according to previously described methods, with slight modifications (Breger et al., 2007; Shivers et al., 2010). After conditioning of $C$. elegans to each probiotic candidate on NGM plates containing nystatin $(5 \mu \mathrm{g} /$ $\mathrm{mL}$ ) for $24 \mathrm{~h}, 20$ worms were exposed to Staphylococcus aureus RN6390 on tryptic soy broth plates, after which live worms were transferred to fresh plates and counted every day. A worm was considered dead when it no longer responded to gentle touch with a platinum wire; dead worms stuck to the walls of the plate were censored from analysis. All C. elegans were cultured at $25^{\circ} \mathrm{C}$. Nematode survival was examined using the Kaplan-Meier method, and differences were determined with the log-rank test (Stata 6; StataCorp LP, College Station, TX).

Thereafter, conventional and traditional in vitro tests, including acid tolerance, bile resistance, bacteriocin activity, cholesterol reduction, and adhesion to in vitro mucin tests were performed on selected probiotic candidates. Susceptibility to low $\mathrm{pH}$ and bile content were assessed as described previously with minor modifications (Oh et al., 2000). The ability of strains to grow at low $\mathrm{pH}$ was evaluated in acidified MRS broth (pH 2.5) containing 250 unit/mg of pepsin (SigmaAldrich, St. Louis, MO). Resistance of the strains to bile (oxgall) was determined in MRS broth containing $0.5 \%$ oxgall (Sigma-Aldrich). Cholesterol assimilation ability of the culture supernatant was determined using o-phthalaldehyde (Rudel and Morris, 1973), and Lactobacillus acidophilus ATCC 43121 and E4191 were used as control strains (Lee et al., 2010). de Man, Rogosa, and Sharpe-thioglycolate broth was prepared daily by supplementation with $0.2 \%$ sodium thioglycolate (Sigma Chemical Co., St. Louis, MO). This broth was further supplemented, when necessary, with $0.2 \%$ taurocholic acid (Sigma Chemical Co.). Cholesterolphosphatidylcholine micelles were generated using a published procedure (Razin et al., 1980). Total cholesterol content of evaporated residues was then determined by colorimetric assay using o-phthalaldehyde. 
(A)

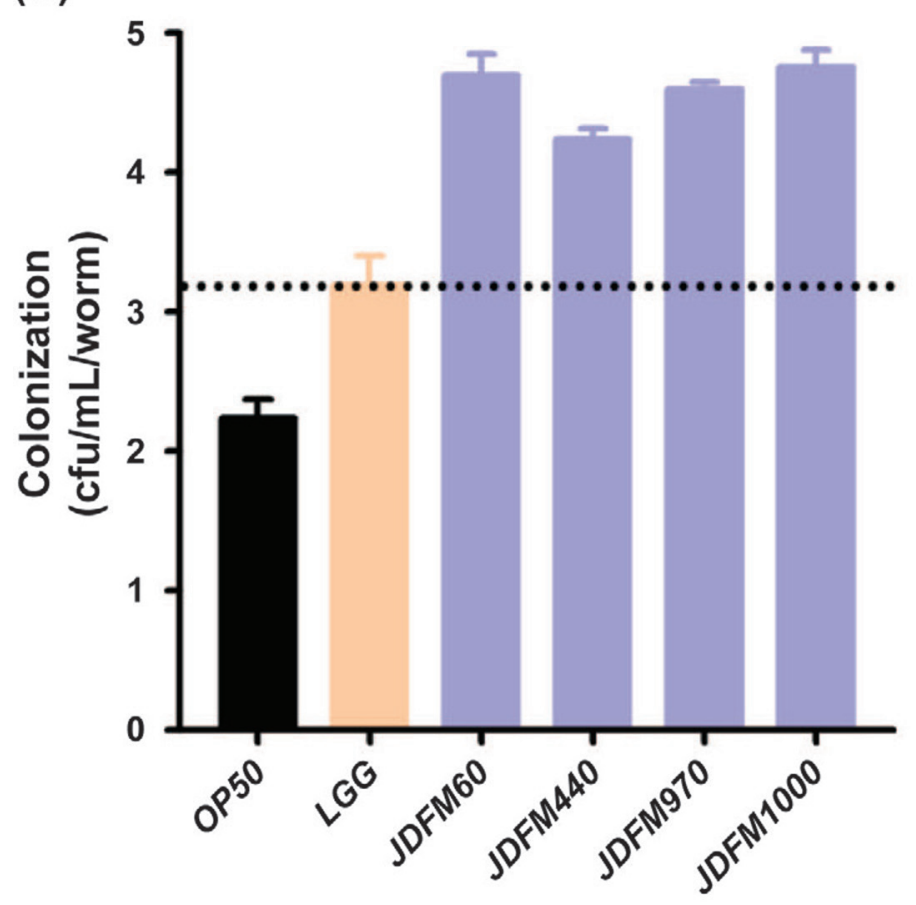

(B)
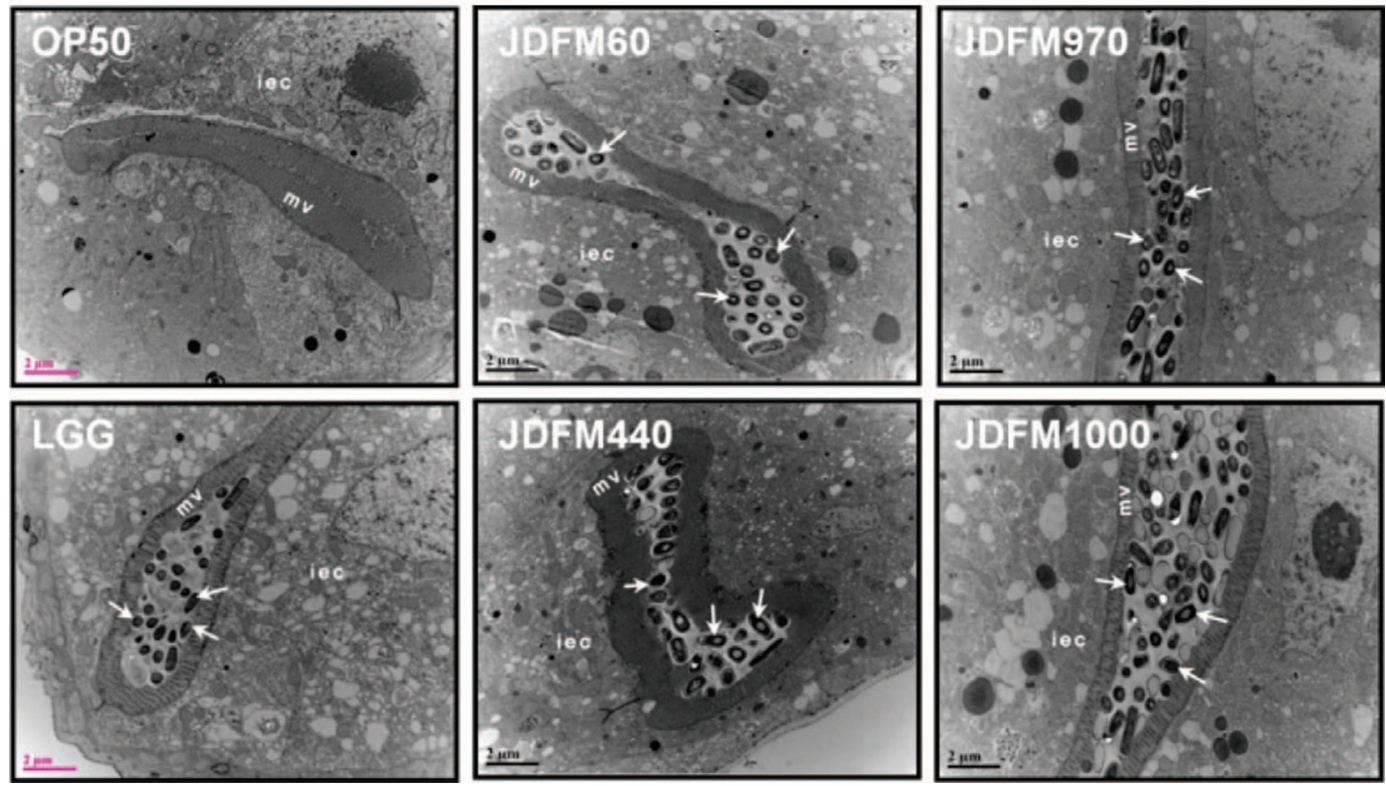

Figure 1. Colonization of the nematode intestine by lactic acid bacteria (LAB) strains. (A) After exposure of nematodes to LAB cells for a period of time, nematodes were washed 5 times with M9 medium and worms were placed in new sterile tubes containing M9 medium with 1\% Triton X-100 and mechanically disrupted using a pestle. Colonization was determined by plating on modified de Man, Rogosa, and Sharpe (MRS; pH 5.0) agar (for LAB strains) or Luria-Bertani agar (for Escherichia coli OP50). Data are expressed as means \pm SD of 3 experiments. (B) Visualization of Caenorhabditis elegans intestine after conditioning with the 4 selected LAB strains for 5 d: transmission electron microscopy micrographs of transversal mid-body sections of animals feeding on nonpathogenic E. coli OP50 (normal foods), Lactobacillus rhamnosus GG (LGG; positive control), and the $4 \mathrm{LAB}$ strains for $7 \mathrm{~d}$; microvilli (mv), intestinal epithelial cells (iec), bacteria were visible in the intestinal lumen (arrows). Scale bars indicate $2 \mu \mathrm{m}$. Color version available in the online PDF. 
Uninoculated sterile broth was analyzed as the negative control. The same procedure was used for the standard curve, except specified amounts of purified cholesterol (Sigma Chemical Co.) were assayed. The ability of the strains to adhere to a mucin surface was investigated using an in vitro mucus layer in the wells of a 96-well plate (Azcarate-Peril et al., 2009), and results were compared with L. rhamnosus GG as a positive control. Attachment ability was determined by counting the number of bacterial cells in the in vitro mucus layer. The significance of differences in bacterial numbers from colony-forming unit counting was determined by Student's $t$-test. Each experiment was performed in triplicate. A $P$-value less than 0.05 for all replicate experiments was considered statistically significant. The final $4 \mathrm{LAB}$ strains selected were identified by $16 \mathrm{~S}$ rRNA sequencing.

Approximately 2,000 LAB stains isolated from infant feces were investigated by assessing in vivo colonization of the intestinal tract, lifespan extension, and resistance to pathogen infection using $C$. elegans as a model. Four strains (JDFM60, JDFM440, JDFM970, and JDFM1000) were selected based on the results of 3 experiments. As shown in Figure 1, these 4 strains were characterized by remarkably high colonization ability. Strains exhibited an outstanding persistence on the $C$. elegans intestine of over $4.3 \mathrm{cfu} / \mathrm{mL}$ per worm; this is higher than the value of $3.2 \mathrm{cfu} / \mathrm{mL}$ per worm obtained for the positive control (L. rhamnosus $\mathrm{GG}$ ), which has been shown to bind to enterocytes in several previous studies (Figure 1A). Consistent with the plate-counting results, transmission electron microscopy images showed that bacterial cells accumulated in the lumen as clumps of cells surrounded by extracellular matrix (Figure 1B). In contrast, E. coli OP50-fed animals did not have clumps of bacterial cells, and had a nondistended intestinal lumen and intestinal epithelial cells filled with lipid droplets. Equally importantly, worms exposed to the $4 \mathrm{LAB}$ strains had a significantly extended lifespan, as shown in Figure 2A. In particular, JDFM60 notably prolonged the average lifespan compared with worms fed $E$. coli OP50 (normal feed for $C$. elegans). These results indicate that the $4 \mathrm{LAB}$ strains induced resistance to aging in C. elegans. Furthermore, we evaluated the effect of LAB strain conditioning on nematode infection with the foodborne pathogen $S$. aureus; worms fed E. coli OP50 served as a negative control (Figure 2B). As expected, worms were killed by exposure to $S$. aureus RN6390 within $7 \mathrm{~d}$ after transfer to a lawn of this pathogen. Interestingly, conditioning with $4 \mathrm{LAB}$ strains for $24 \mathrm{~h}$ significantly enhanced the resistance of nematodes exposed to $S$. aureus RN6390. Taken together, the 4 selected LAB strains had host health-promoting effects, including longevity and re- sistance to pathogen infection, in addition to showing excellent colonization of the worm intestinal tract.

Next, we assessed the 4 selected strains using conventional and traditional in vitro methods (Figure $3)$. Tolerance to low $\mathrm{pH}$ and bile salt, which reflect conditions in the digestive tracts, are fundamental to probiotic activity. To examine the ability of the 4 selected LAB strains to survive acidic conditions $(\mathrm{pH}$ 2.5 ), which would enable them to survive in the large intestine of humans, isolates were incubated for $3 \mathrm{~h}$ at $37^{\circ} \mathrm{C}$ with $1,000 \mathrm{U}$ of pepsin $/ \mathrm{mL}$ and assayed by viable cell counting. Strain JDFM440 had a higher survival rate than the other isolates, and was followed by JDFM60, JDFM970, and JDFM1000. However, no difference existed in the survival of these strains and the positive control; the survival rate of all strains was approximately $100 \%$. Selected strains were also examined for their ability to grow at concentrations of $0.5 \%$ bile salt for $24 \mathrm{~h}$. Survival rate of the positive control and the 4 tested strains was approximately 100\%. Taken together, the isolates demonstrated strong survival under acid and bile conditions (Figure 3A and B). In addition, 4 selected strains were tested for their ability to reduce cholesterol in vitro in the presence of bile salts (Figure 3C). The amount of cholesterol assimilated ranged from 75 to $83 \%$. Strain JDFM60 assimilated the most cholesterol. Moreover, to compare the in vivo colonization assay, we examined the ability of the selected strains to attach to abiotic surfaces using an in vitro mucin-binding assay. Tested strains adhered strongly to the mucin surface $(\geq 5.4 \log \mathrm{cfu} /$ $\mathrm{mL}$ ), although they exhibited lower adherence than the positive control, L. rhamnosus GG (Figure 3D). Thus, the 4 LAB strains selected by our direct in vivo methods also showed characteristic probiotic properties via in vitro biological assays. All isolates were identified as Lactobacillus plantarum using $16 \mathrm{~S}$ rRNA sequencing analysis.

In recent years, $C$. elegans has been used to investigate the health-promoting activity of probiotic bacteria such as Lactobacillus and Bifidobacterium as well as to investigate the pathogenesis of foodborne pathogens such as $S$. aureus, Salmonella spp., and Pseudomonas aeruginosa. Other researchers (Ikeda et al., 2007) have reported that feeding worms specific bifidobacteria resulted in an increased average lifespan compared with that of worms fed E. coli OP50, with lifespan extension of between 17 and 33\%. In addition, we reported that conditioning nematodes with the probiotic bacterium L. acidophilus NCFM resulted in significantly prolonged host survival after exposure to pathogenic strains of Enterococcus faecalis and $S$. aureus, including multidrug-resistant isolates (Kim and Mylonakis, 2012). In the present study, we 
(A)
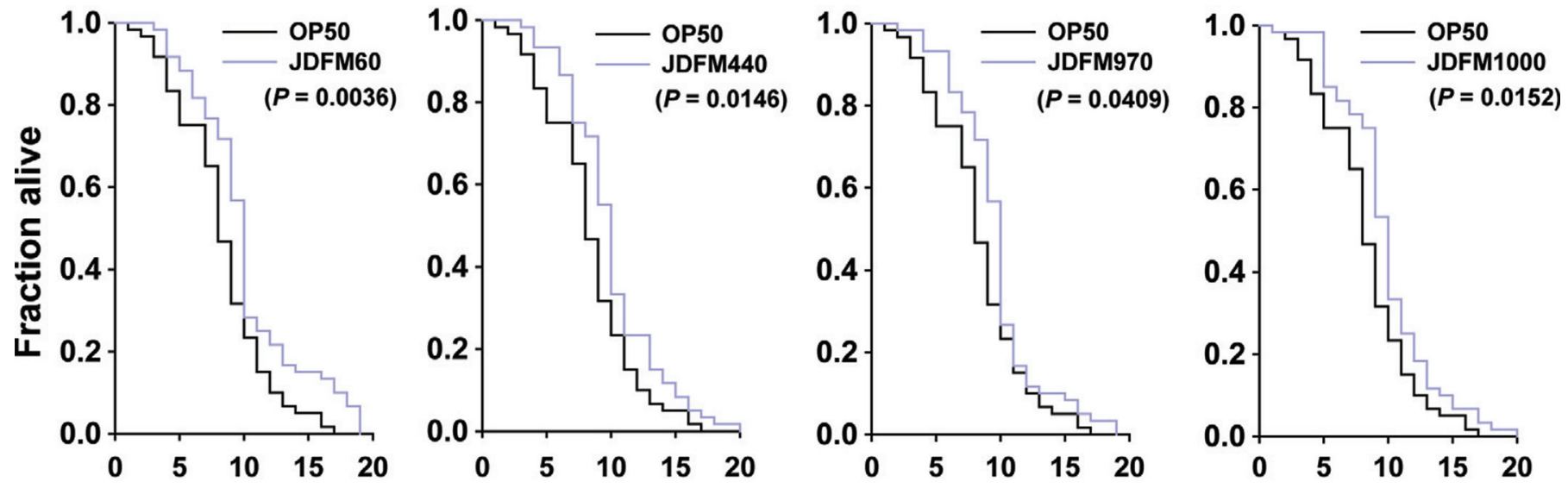

(B)
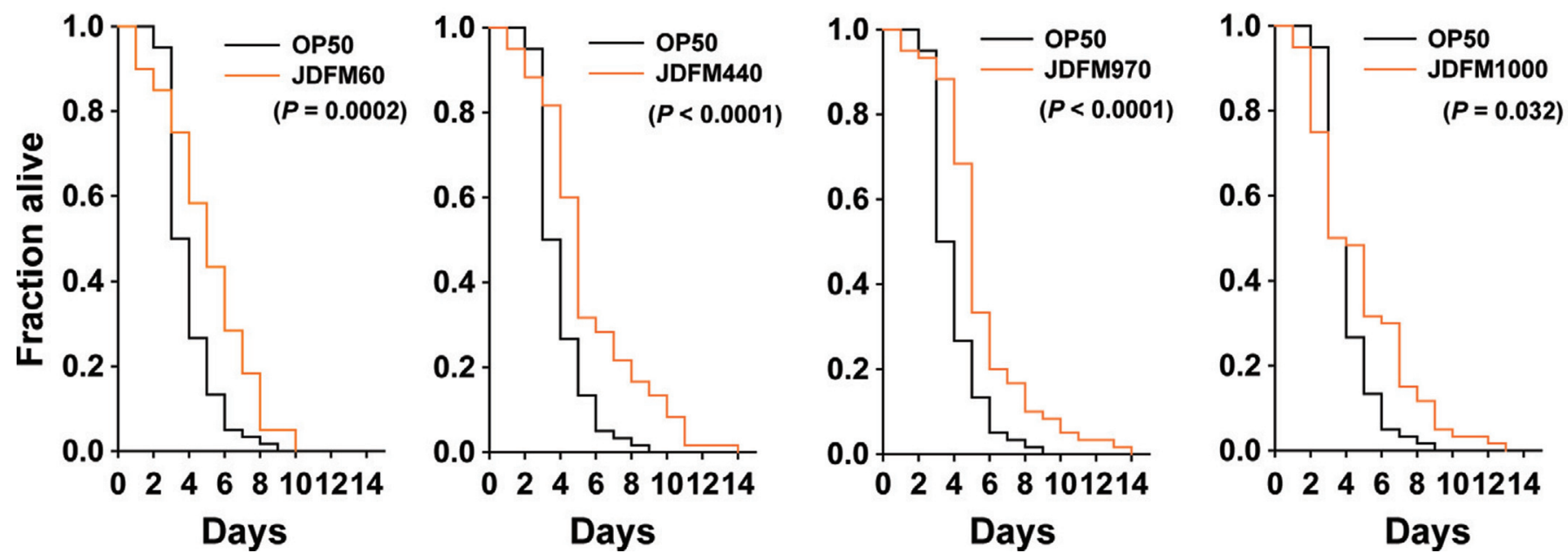

Figure 2. Effects of lactic acid bacteria (LAB) strains on lifespan and resistance to Staphylococcus aureus infection in Caenorhabditis elegans. (A) Lifespan curves of L4-stage C. elegans fer-15; fem-1 fed JDFM60, JDFM440, JDFM970, JDFM1000, or Escherichia coli OP50. Survival statistics: $P=0.0036$ for JDFM60, $P=0.015$ for JDFM440, $P=0.041$ for JDFM970, and $P=0.015$ for JDFM1000, respectively, compared with worms exposed to E. coli strain OP50. (B) Survival of $C$. elegans fer-15; fem-1 infected with $S$. aureus strain RN6390 after conditioning with the 4 selected LAB strains for $24 \mathrm{~h}$. Survival statistics: $P=0.0037$ for JDFM60, $P=0.0013$ for JDFM440, $P=0.0003$ for JDFM970, and $P$ $=0.032$ for JDFM1000, respectively, compared with worms exposed to E. coli strain OP50 for $24 \mathrm{~h}$. Color version available in the online PDF.

used C. elegans as a direct and high-throughput in vivo tool for probiotic screening and identification. Newly isolated Lactobacillus spp. were also studied in terms of their effect on C. elegans longevity. We found that $\mathrm{LAB}$ isolated strains from infant feces were able to colonize the intestinal tract of $C$. elegans, prolong lifespan, and enhance the resistance of the host to pathogen infection. Although further in vivo corroboration is required, our results indicate that LAB feeding stimulates the innate immune response via the lumen of the C. elegans intestine. Further research should focus on the mechanisms by which the probiotics exert their antiaging and immune enhancement effects in $C$. elegans. The 4 strains satisfied all requirements as a useful probiotic candidate. In addition, they survived in gastric juice, tolerated bile salt, and adhered to the mucin surface.

In conclusion, we demonstrated that $C$. elegans is a promising preclinical model for selecting and screening potential probiotics; our screening system allows the host health-promoting activity of potential probiotics to be assessed in a faster, simpler, and more effective way than conventional in vitro and in vivo approaches. The LAB strains that we identified show promise for use as probiotic agents, feed additives, and in dairy food applications. 
(A)

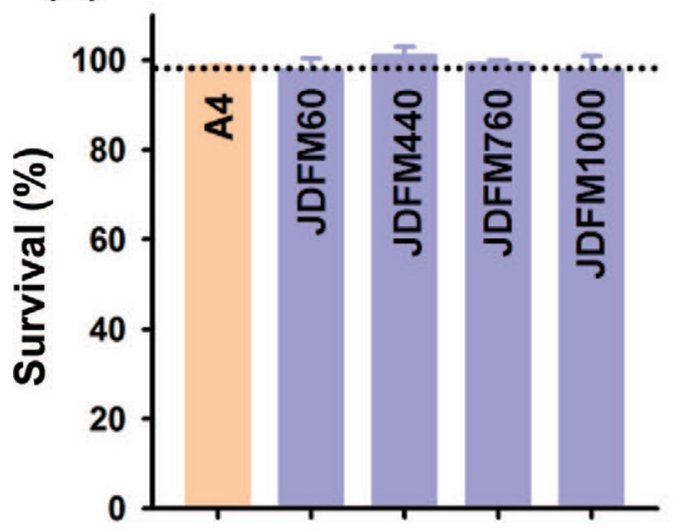

(C)

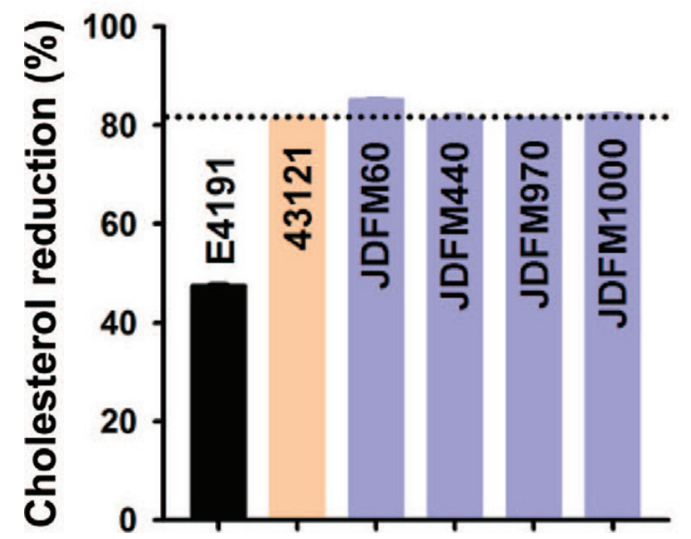

(B)

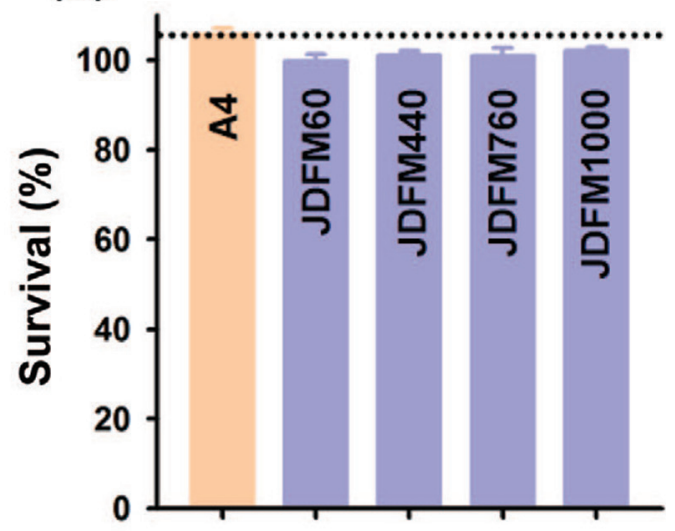

(D)

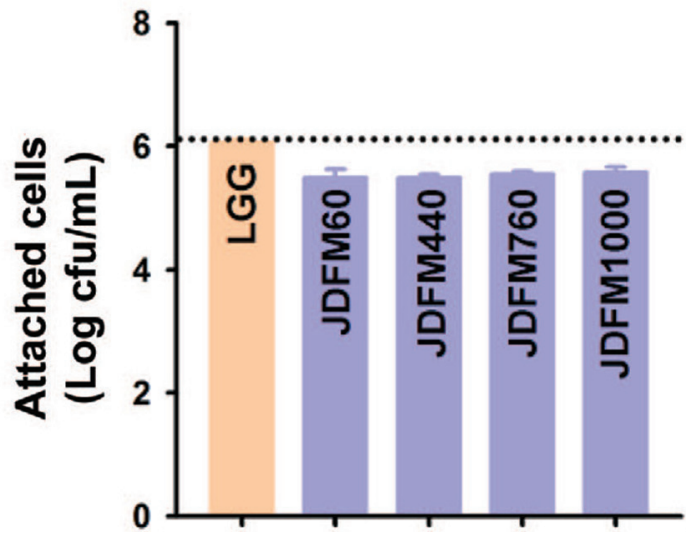

Figure 3. Conventional and traditional in vitro assay results for the 4 selected strains (JDFM60, JDFM440, JDFM970, and JDFM1000). Susceptibility of the 4 selected lactic acid bacteria (LAB) strains to (A) synthetic gastric juice (pH 2.5) and (B) bile conditions with $0.5 \%$ oxgall as well as their ability for $(\mathrm{C})$ cholesterol reduction and (D) attachment on a mucus layer in vitro. Data are expressed as means \pm SD of 3 experiments. A4 = Lactobacillus acidophilus A4; E4191 = L. acidophilus ATCC E4191; 43121 = L. acidophilus ATCC 43121; LGG = Lactobacillus rhamnosus GG. Color version available in the online PDF.

\section{ACKNOWLEDGMENTS}

This research was supported by the Basic Science Research Program through the National Research Foundation of Korea (NRF; Seoul, Korea) funded by the Ministry of Science, Information and Communications Technology (ICT) and Future Planning (Gwacheon, Korea; 2014R1A1A1006872).

\section{REFERENCES}

Aballay, A., P. Yorgey, and F. M. Ausubel. 2000. Salmonella typhimurium proliferates and establishes a persistent infection in the intestine of Caenorhabditis elegans. Curr. Biol. 10:1539-1542.

Azcarate-Peril, M. A., R. Tallon, and T. R. Klaenhammer. 2009. Temporal gene expression and probiotic attributes of Lactobacillus acidophilus during growth in milk. J. Dairy Sci. 92:870-886.

Breger, J., B. B. Fuchs, G. Aperis, T. I. Moy, F. M. Ausubel, and E. Mylonakis. 2007. Antifungal chemical compounds identified using a C. elegans pathogenicity assay. PLoS Pathog. 3:e18.

Brenner, S. 1974. The genetics of Caenorhabditis elegans. Genetics 77:71-94.
Buchanan, R. E., and N. E. Gibbons, editors. 1974. Bergey's Manual of Determinative Bacteriology. 8th ed. The Williams and Wilkins Co., Baltimore, MD

du Toit, M., C. M. A. P. Franz, L. M. T. Dicks, U. Schillinger, P. Haberer, B. Warlies, F. Ahrens, and W. H. Holzapfel. 1998. Characterisation and selection of probiotic lactobacilli for a preliminary minipig feeding trial and their effect on serum cholesterol levels, faeces $\mathrm{pH}$ and faeces moisture content. Int. J. Food Microbiol. 40:93-104.

Duary, R. K., Y. S. Rajput, V. K. Batish, and S. Grover. 2011. Assessing the adhesion of putative indigenous probiotic lactobacilli to human colonic epithelial cells. Indian J. Med. Res. 134:664-671.

FAO/WHO (Food and Agriculture Organization of the United Nations/World Health Organization). 2002. Guidelines for the evaluation of probiotics in food. Food and Agriculture Organization of the United Nations and World Health Organization Working Group Report. Accessed July 20, 2011. http://www.who.int/ foodsafety/fs_management/en/probiotic_guidelines.pdf.

Fuller, R. 1989. Probiotics in man and animals. J. Appl. Bacteriol. $66: 365-378$.

Garigan, D., A.-L. Hsu, A. G. Fraser, R. S. Kamath, J. Ahringer, and C. Kenyon. 2002. Genetic analysis of tissue aging in Caenorhabditis elegans: A role for heat-shock factor and bacterial proliferation. Genetics 161:1101-1112. 
Garsin, D. A., C. D. Sifri, E. Mylonakis, X. Qin, K. V. Singh, B. E. Murray, S. B. Calderwood, and F. M. Ausubel. 2001. A simple model host for identifying gram-positive virulence factors. Proc. Natl. Acad. Sci. USA 98:10892-10897.

Guo, X.-H., J.-M. Kim, H.-M. Nam, S.-Y. Park, and J.-M. Kim. 2010 Screening lactic acid bacteria from swine origins for multistrain probiotics based on in vitro functional properties. Anaerobe $16: 321-326$.

Heller, K. J. 2001. Probiotic bacteria in fermented foods: Product characteristics and starter organisms. Am. J. Clin. Nutr. 73:374S379S.

Ikeda, T., C. Yasui, K. Hoshino, K. Arikawa, and Y. Nishikawa. 2007. Influence of lactic acid bacteria on longevity of Caenorhabditis elegans and host defense against Salmonella enterica serovar Enteritidis. Appl. Environ. Microbiol. 73:6404-6409.

Kim, Y., and E. Mylonakis. 2012. Caenorhabditis elegans immune conditioning with the probiotic bacterium Lactobacillus acidophilus strain NCFM enhances gram-positive immune responses. Infect. Immun. 80:2500-2508.

Lamari, F., K. Sadok, A. Bakhrouf, and F.-J. Gatesoupe. 2014. Selection of lactic acid bacteria as candidate probiotics and in vivo test on Artemia nauplii. Aquacult. Int. 22:699-709.

Lee, J., Y. Kim, H. S. Yun, J. G. Kim, S. Oh, and S. H. Kim. 2010 Genetic and proteomic analysis of factors affecting serum cholesterol reduction by Lactobacillus acidophilus A4. Appl. Environ. Microbiol. 76:4829-4835.

Leung, M. C. K., P. L. Williams, A. Benedetto, C. Au, K. J. Helmcke, M. Aschner, and J. N. Meyer. 2008. Caenorhabditis elegans: An emerging model in biomedical and environmental toxicology. Toxicol. Sci. 106:5-28.
McGhee, J. D. 2007. The C. elegans intestine. WormBook 27:1-36.

Oh, S., S. Kim, and R. Worobo. 2000. Characterization and purification of a bacteriocin produced by a potential probiotic culture, Lactobacillus acidophilus 30SC. J. Dairy Sci. 83:2747-2752.

Ouwehand, A. C., P. V. Kirjavainen, M.-M. Grönlund, E. Isolauri, and S. J. Salminen. 1999. Adhesion of probiotic micro-organisms to intestinal mucus. Int. Dairy J. 9:623-630.

Razin, S., S. Kutner, H. Efrati, and S. Rottem. 1980. Phospholipid and cholesterol uptake by Mycoplasma cells and membranes. Biochim. Biophys. Acta 598:628-640.

Rudel, L. L., and M. D. Morris. 1973. Determination of cholesterol using o-phthalaldehyde. J. Lipid Res. 14:364-366.

Sanders, M. E. 2008. Probiotics: Definition, sources, selection, and uses. Clin. Infect. Dis. 46:S58-S61.

Shivers, R. P., D. J. Pagano, T. Kooistra, C. E. Richardson, K. C Reddy, J. K. Whitney, O. Kamanzi, K. Matsumoto, N. Hisamoto, and D. H. Kim. 2010. Phosphorylation of the conserved transcription factor ATF-7 by PMK-1 p38 MAPK regulates innate immunity in Caenorhabditis elegans. PLoS Genet. 6:e1000892.

Sugimura, Y.. T. Hagi, and T. Hoshino. 2011. Correlation between in vitro mucus adhesion and the in vivo colonization ability of lactic acid bacteria: Screening of new candidate carp probiotics. Biosci. Biotechnol. Biochem. 75:511-515.

Turchi, B., S. Mancini, F. Fratini, F. Pedonese, R. Nuvoloni, F. Bertelloni, V. V. Ebani, and D. Cerri. 2013. Preliminary evaluation of probiotic potential of Lactobacillus plantarum strains isolated from Italian food products. World J. Microbiol. Biotechnol. 29:19131922 